\title{
PROOF OF A CONJECTURE OF ERDÖS ABOUT THE LONGEST POLYNOMIAL
}

\author{
B. D. BOJANOV
}

\begin{abstract}
In $1939 \mathrm{P}$. Erdös conjectured that the Chebyshev polynomial $T_{n}(x)$ has a maximal arc-length in $[-1,1]$ among the polynomials of degree $n$ which are bounded by 1 in $[-1,1]$. We prove this conjecture for every natural $n$.
\end{abstract}

1. Introduction. P. Erdös proved in [2] that the function $\cos n t$ has a maximal arc-length in $[-\pi, \pi]$ among all trigonometric polynomials of order $n$ with a uniform norm equal to 1 . He has conjectured that the Chebyshev polynomial

$$
T_{n}(x)=\cos (n \arccos x), \quad-1 \leqslant x \leqslant 1,
$$

is the unique extremal function in the corresponding analogous problem in the set $\pi_{n}$ of algebraic polynomials of degree less than or equal to $n$.

Denote by $l(f)$ the arc-length of the function $f$ in $[-1,1]$, i.e.,

$$
l(f):=\int_{-1}^{1}\left[1+f^{\prime 2}(x)^{2}\right]^{1 / 2} d x .
$$

Set $\|f\|=\max \{|f(x)|:-1 \leqslant x \leqslant 1\}$.

CONJECTURE OF ERDös. The quantity

$$
\sup \left\{l(f): f \in \pi_{n},\|f\| \leqslant 1\right\} \quad(n=1,2, \ldots)
$$

is attained if and only if $f= \pm T_{n}$.

This conjecture has remained an open problem for over 40 years. In a recent work Szabados [4] showed that the polynomials $T_{n}$ are asymptotically extremal as $n \rightarrow \infty$. We prove here the conjecture of Erdös for each natural number $n$. Our proof is based on a variational approach.

2. Explanatory statement. The problem of Erdös is set for the domain $[-1,1] \times$ $[-1,1]$, i.e., for the class of polynomials $f \in \pi_{n}$ such that $|f(x)|<1$ if $|x|<1$. One may guess that the solution $f(x)$ in this particular case suffices to construct the solution $f(M ; x)$ of the corresponding problem about the longest polynomial in the domain $[-1,1] \times[-M, M]$ for every $M>0$. One even suggests the following simple formula:

$$
f(M ; x)=M f(x) .
$$

It turns out (see Theorem 1) that (*) is actually true. But this is not evident. The problem (*) is as difficult as that of Erdös. In any case, the relatioin (*) yields easily the conjecture of Erdös. Indeed, suppose that (*) holds for every $M>0$.

Received by the editors January 13, 1981.

1980 Mathematics Subject Classification. Primary 41A17, 49A27.

Key words and phrases. Arc-length, Chebyshev polynomial, extremal problem for polynomials. 
Then

$$
\frac{1}{M} \int_{-1}^{1}\left[1+M^{2} g^{\prime 2}(x)\right]^{1 / 2} d x \leqslant \frac{1}{M} \int_{-1}^{1}\left[1+M^{2} f^{\prime 2}(x)\right]^{1 / 2} d x
$$

for each $M>0$, provided $g \in \pi_{n}$, and $\|g\| \leqslant 1$. If we let $M$ tend to infinity, we get $\int_{-1}^{1}\left|g^{\prime}(x)\right| d x \leqslant \int_{-1}^{1}\left|f^{\prime}(x)\right| d x$. Thus, $f$ should have a maximal variation in $[-1,1]$. Therefore $f= \pm T_{n}$.

Finally, note that the problem on an arbitrary interval $[a, b]$ is easily reduced to the problem on $[-1,1]$ by a linear transformation.

3. Main result. In what is to follow, let $M$ be a fixed positive number. With every natural number $n$ we associate the set $\Omega_{n} \subset \pi_{n}$ which is defined as follows. The polynomial $f \in \pi_{n}$ belongs to $\Omega_{n}$ if there exist $m+1$ points $\left\{x_{i}\right\}_{0}^{m}$ ( $m \in$ $\{1, \ldots, n\})$ such that

$$
\begin{gathered}
-1=x_{0}<x_{1}<\cdots<x_{m-1}<x_{m}=1, \\
\left|f\left(x_{i}\right)\right|=M, \quad i=0, \ldots, m, \\
f\left(x_{i}\right)=-f\left(x_{i+1}\right), \quad i=0, \ldots, m-1
\end{gathered}
$$

and $f(x)$ is a monotone function in $\left[x_{i}, x_{i+1}\right], i=0, \ldots, m-1$. It is clear that $\|f\|=M$ if $f \in \Omega_{n}$.

The basic idea of our proof is presented in the following lemma.

LeMma 1. Suppose that $f \in \pi_{n},\|f\|=M$ and

$$
l(f)=\sup \left\{l(g): g \in \pi_{n},\|g\| \leqslant M\right\} \text {. }
$$

Then $f \in \Omega_{n}$.

Proof. Without loss of generality we assume that $f(x)>0$ for each sufficiently large $x>0$. Denote by $\left\{x_{i}\right\}_{1}^{m-1}$ the distinct zeros of $f^{\prime}(x)$ in $(-1,1)$. Obviously $m \leqslant n$. Set, for convenience, $x_{0}=-1, x_{m}=1, \omega(x)=f^{\prime}(x)$. We shall show that

$$
f\left(x_{i}\right)=(-1)^{m-1} M, \quad i=0, \ldots, m \text {. }
$$

This implies that $f \in \Omega_{n}$.

Introduce the polynomials

$$
g_{i}(x)=\left(x^{2}-1\right) \omega(x) /\left(x-x_{i}\right), \quad i=0, \ldots, m .
$$

We intend to estimate the arc-length $\sigma_{i}(\varepsilon):=l\left(f+\varepsilon g_{i}\right)$ for small $\varepsilon$. Our first task is to show that

$$
\sigma_{i}^{\prime}(0)>0
$$

for $i=0, \ldots, m$. It is seen that

$$
\sigma_{i}^{\prime}(0)=\int_{-1}^{1} \frac{\omega(x)}{\left[1+\omega^{2}(x)\right]^{1 / 2}} g_{i}^{\prime}(x) d x
$$

In the case $i=0$ a straightforward calculation gives

$$
\sigma_{0}^{\prime}(0)=2\left[1+\omega^{2}(-1)\right]^{1 / 2}-\int_{-1}^{1}\left[1+\omega^{2}(x)\right]^{-1 / 2} d x>0
$$


Similarly, $\sigma_{m}^{\prime}(0)>0$. Now suppose that $1 \leqslant i \leqslant m-1$. Integrating by parts, we get

$$
\sigma_{i}^{\prime}(0)=\int_{-1}^{1} \frac{x^{2}-1}{x-x_{i}}\left\{\left[1+\omega^{2}(x)\right]^{-1 / 2}\right\}^{\prime} d x
$$

The integrand is a continuous function in $[-1,1]$. Therefore $\sigma_{i}^{\prime}(0)<\infty$ and $\sigma_{i}^{\prime}(0)=$ $\lim \left\{\sigma_{i}(\delta): \delta \rightarrow 0\right\}$ where

$$
\sigma_{i}(\delta)=\int_{\Omega(\delta)} \frac{x^{2}-1}{x-x_{i}}\left\{\left[1+\omega^{2}(x)\right]^{-1 / 2}\right\}^{\prime} d x
$$

and $\Omega(\delta):=\left[-1, x_{i}-\delta\right] \cup\left[x_{i}+\delta, 1\right]$. Next we calculate $\mathcal{T}_{i}(\delta)$. Observe first that $\omega\left(x_{i} \pm \delta\right)=O(\delta)$. This yields, for instance, by Taylor's formula, that

$$
\left[1+\omega^{2}\left(x_{i} \pm \delta\right)\right]^{-1 / 2}=1+O\left(\delta^{2}\right) .
$$

Further, by the mean-value theorem for integrals, there exist points $\xi_{1}=\xi_{1}(\delta) \in$ $\left[-1, x_{i}-\delta\right]$ and $\xi_{2}=\xi_{2}(\delta) \in\left[x_{i}+\delta, 1\right]$ such that

$$
\begin{gathered}
\int_{-1}^{x_{i}-\delta}\left[1+\omega^{2}(x)\right]^{-1 / 2}\left(x-x_{i}\right)^{-2} d x=c_{1}(\delta)\left[1 / \delta-1 /\left(1+x_{i}\right)\right], \\
\int_{x_{i}+\delta}^{1}\left[1+\omega^{2}(x)\right]^{-1 / 2}\left(x-x_{i}\right)^{-2} d x=c_{2}(\delta)\left[1 / \delta-1 /\left(1-x_{i}\right)\right]
\end{gathered}
$$

where $c_{j}(\delta)=\left[1+\omega^{2}\left(\xi_{j}\right)\right]^{-1 / 2}, j=1,2$. Obviously

$$
0<c_{j}(\delta) \leqslant 1, \quad j=1,2 .
$$

Let us set, for convenience, $A(\delta)=\int_{\Omega(\delta)}\left[1+\omega^{2}(x)\right]^{-1 / 2} d x$. Now, taking into account the relations (3) and (4), after integration by parts, we obtain

$$
\begin{aligned}
\mathscr{T}_{i}(\delta)= & {\left.\left[\left(x^{2}-1\right) /\left(x-x_{i}\right)\right]\left[1+\omega^{2}(x)\right]^{-1 / 2}\right|_{x_{i}+\delta} ^{x_{i}+\delta} } \\
& -\int_{\Omega(\delta)}\left[1+\omega^{2}(x)\right]^{-1 / 2}\left\{1+\left(1-x_{i}^{2}\right) /\left(x-x_{i}\right)^{2}\right\} d x \\
= & \delta^{-1}\left[c_{1}(\delta)+c_{2}(\delta)-2\right]\left(x_{i}^{2}-1\right)+O(\delta)-A(\delta) \\
& -c_{1}(\delta)\left(x_{i}-1\right)+c_{2}(\delta)\left(x_{i}+1\right) .
\end{aligned}
$$

But, as we have already mentioned, $\mathscr{T}_{i}(\delta)$ has a limit as $\delta \rightarrow 0$. Then $c_{1}(\delta)+c_{2}(\delta)$ must tend to 2, which combined with (5) implies $c_{j}(\delta) \rightarrow 1, j=1,2$, as $\delta \rightarrow 0$. Moreover, $c_{j}(\delta)=1-\alpha_{j} \delta+o(\delta), j=1,2$, with some constants $\alpha_{j} \geqslant 0$. Therefore

$$
\sigma_{i}^{\prime}(0)=\lim \left\{\mathscr{T}_{i}(\delta): \delta \rightarrow 0\right\}=-A(0)+2-\left(\alpha_{1}+\alpha_{2}\right)\left(x_{i}^{2}-1\right)>0 .
$$

Our claim (2) is proved.

Now, let us assume that $f$ does not belong to $\Omega_{n}$. Then there exists at least one point $x_{i} \in\left\{x_{0}, \ldots, x_{m}\right\}$ such that $\left|f\left(x_{i}\right)\right|<M$. Consider the polynomial $\varphi_{\varepsilon}(x):=f(x)+\varepsilon g_{i}(x)$. Evidently, $l\left(\varphi_{\varepsilon}\right)=\sigma_{i}(\varepsilon)=\sigma_{i}(0)+\varepsilon \sigma_{i}^{\prime}\left(t_{\varepsilon}\right)=l(f)+\varepsilon \sigma_{i}^{\prime}\left(t_{e}\right)$ where $0<t_{\varepsilon}<\varepsilon$. But, according to (2), there eixsts an $\varepsilon_{0}>0$ such that $\sigma:=\min \left\{\sigma_{i}^{\prime}(t): 0 \leqslant t \leqslant \varepsilon_{0}\right\}>0$. Therefore

$$
l\left(\varphi_{\varepsilon}\right) \geqslant l(f)+\sigma \varepsilon
$$

for each $\varepsilon \in\left[0, \varepsilon_{0}\right]$. 
Let us estimate the uniform norm of $\varphi_{e}$ in $[-1,1]$ for small $\varepsilon$. In order to do this, it suffices to investigate the function $\varphi_{\varepsilon}(x)$ near the points $\left\{x_{j}\right\}$ for which $\left|f\left(x_{j}\right)\right|=$ $M$. Let $x_{k}$ be such a point. Without loss of generality we may assume that $f\left(x_{k}\right)=M$. Suppose that $h$ is chosen to satisfy the requirement $x_{j} \notin\left[x_{k}-h, x_{k}+\right.$ $h] \cap[-1,1]=: B\left(x_{k} ; h\right)$ for every $j \neq k$. Let $\varphi_{e}(x)$ attain its maximal value in the neighbourhood $B\left(x_{k} ; h\right)$ of $x_{k}$ at the point $z_{k}(\varepsilon)$. On expanding $\varphi_{e}(x)$ in a partial Taylor series around $x=x_{k}$, we get

$$
\varphi_{\varepsilon}\left(z_{k}(\varepsilon)\right) \leqslant M+\varepsilon\left\|g_{i}^{\prime}\right\|\left|z_{k}(\varepsilon)-x_{k}\right|
$$

for sufficiently small $\varepsilon>0$. It is not difficult to see that $\left|z_{k}(\varepsilon)-x_{k}\right| \rightarrow 0$ as $\varepsilon \rightarrow 0$. Then, in view of the last inequality, $\left\|\varphi_{e}\right\|<M+\varepsilon \delta(\varepsilon)$, where $\delta(\varepsilon)$ is a function which tends to zero as $\varepsilon \rightarrow 0$. Now construct the polynomial

$$
\psi_{\varepsilon}(x)=\left(1-\frac{\varepsilon \delta(\varepsilon)}{M+\varepsilon \delta(\varepsilon)}\right) \varphi_{\varepsilon}(x)
$$

Clearly, $\psi_{e} \in \pi_{n}$ and $\left\|\psi_{e}\right\|<M$. We shall show that $l\left(\psi_{e}\right)>l(f)$ for small $\varepsilon>0$. Indeed, since $L:=\partial l(\lambda f) /\left.\partial \lambda\right|_{\lambda=1}>0$, we have $l\left(\psi_{\varepsilon}\right)>l\left(\varphi_{e}\right)-(2 L / M) \varepsilon \delta(\varepsilon)$ for small $\varepsilon>0$. Next we apply (6) and get

$$
l\left(\psi_{\varepsilon}\right)>l(f)+[\sigma-(2 L / M) \delta(\varepsilon)] \varepsilon>l(f)
$$

for sufficiently small $\varepsilon>0$. Thus, $f$ is not extremal, a contradiction. Therefore $\left|f\left(x_{i}\right)\right|=M$ for $i=0, \ldots, m$. Since $\left\{x_{i}\right\}_{1}^{m-1}$ are all distinct zeros of $f^{\prime}(x)$ in $(-1,1)$, we conclude that $(1)$ is valid. The lemma is proved.

It remains to show that the extremal polynomial $f$ must have $n+1$ points of alternation. For this, we give below an interesting property of the Chebyshev polynomial $T_{n}(x)$.

Let $\left\{\theta_{k}\right\}_{0}^{n}$ be the extremal points of $T_{n}(x)$ in $[-1,1]$. It is well known (see Rivlin [3]) that $\theta_{0}=-1, \theta_{n}=1$ and $T_{n}\left(\theta_{k}\right)=(-1)^{n-k}, k=0, \ldots, n$. Suppose that $f \in \Omega_{n}$ and $f^{\prime}(x)$ has $m-1$ distinct zeros $x_{1}, \ldots, x_{m-1}$ in $(-1,1)$. Evidently, there is an $i \in\{0, \ldots, m-1\}$ such that $x_{i}<0<x_{i+1}$. Consider the partition of $[-1,1]$ into subintervals $\left[x_{0}, x_{1}\right], \ldots,\left[x_{i}, 0\right],\left[0, x_{i+1}\right], \ldots,\left[x_{m-1}, x_{m}\right]$ which we denote, for simplicity, by $I_{0}, \ldots, I_{m}$, respectively. Define the points $t_{1}$ and $t_{2}$ by the conditions

$$
\begin{aligned}
t_{1} \in\left[\theta_{i}, \theta_{i+1}\right], & M T_{n}\left(t_{1}\right)=f(0), \\
t_{2} \in\left[\theta_{i+n-m}, \theta_{i+n-m+1}\right], & M T_{n}\left(t_{2}\right)=f(0) .
\end{aligned}
$$

Denote the intervals $\left[\theta_{0}, \theta_{1}\right], \ldots,\left[\theta_{i}, t_{1}\right],\left[t_{2}, \theta_{i+n-m+1}\right], \ldots,\left[\theta_{n-1}, \theta_{n}\right]$ by $I_{0}^{*}, \ldots, I_{m}^{*}$. We shall refer to $I_{k}^{*}$ as the corresponding interval to $I_{k}$.

LEMMA 2. Suppose that $f$ is a polynomial from the set $\Omega_{n}$ with $m+1$ extremal points, $\alpha \in(-M, M)$ and $k \in\{0, \ldots, m\}$. Let the points $\xi$ and $\eta$ satisfy the conditions

$$
\xi \in I_{k}^{*}, \quad M T_{n}(\xi)=\alpha, \quad \eta \in I_{k}, \quad f(\eta)=\alpha .
$$

Then $\left|f^{\prime}(\eta)\right| \leqslant M\left|T_{n}^{\prime}(\xi)\right|$.

The assertion follows easily from a known extremal property of $\cos n t$. The proof is given with details in [1]. 
We are now prepared to prove the main theorem.

THEOREM 1. Let $n$ be an arbitrary natural number. Then, for each $M>0$, the quantity

$$
\sup \left\{l(f): f \in \pi_{n},\|f\| \leqslant M\right\}
$$

is attained if and only if $f= \pm M T_{n}$.

Proof. Note first that the inequality $|d| \leqslant|c|$ implies

$$
\left(1+c^{2}\right)^{1 / 2} \leqslant\left(1+d^{2}\right)^{1 / 2}+|c|-|d| \text {. }
$$

We shall make use of this in the sequel. Suppose that $f \in \Omega_{n}$ and $[-1,1]=I_{0}$ $\cup \cdots \cup I_{m}$ is the partition of $[-1,1]$ induced by $f$. Let the intervals $I=\left[z_{1}, z_{2}\right]$ and $I^{*}=\left[z_{1}^{*}, z_{2}^{*}\right]$ be corresponding. Denote by $u(y)$ and $v(y)$ the inverse functions of $f(x)$ and $M T_{n}(x)$ in $I$ and $I^{*}$, respectively. According to Lemma 2, we have $\left|v^{\prime}(y)\right| \leqslant\left|u^{\prime}(y)\right|$ for each $y \in(-M, M)$. Then, applying (7), we get

$$
\int_{-M}^{M}\left[1+u^{\prime 2}(y)\right]^{1 / 2} d y \leqslant \int_{-M}^{M}\left[1+v^{\prime 2}(y)\right]^{1 / 2} d y+\int_{-M}^{M}\left|u^{\prime}(y)\right| d y-\int_{-M}^{M}\left|v^{\prime}(y)\right| d y .
$$

Denote by $l(g ; K)$ the arc-length of $g$ over the set $K$. Then the above inequality means that $l(f ; I) \leqslant l\left(M T_{n} ; I^{*}\right)+\left|z_{2}-z_{1}\right|-\left|z_{2}^{*}-z_{1}^{*}\right|$. Summing for $I=$ $I_{0}, \ldots, I_{m}$, we obtain

$$
l(f) \leqslant l\left(M T_{n} ;\left[-1, t_{1}\right] \cup\left[t_{2}, 1\right]\right)+t_{2}-t_{1} \leqslant l\left(M T_{n}\right) .
$$

The equality holds if and only if $t_{1}=t_{2}$, i.e., iff $f= \pm M T_{n}$. The theorem is proved.

4. Acknowledgments. The author is grateful to his colleagues $\mathrm{K}$. Ivanov, S. Dodunekov, M. Petkov and V. Todorov for discussions and comments.

\section{REFERENCES}

1. B. D. Bojanov, An extension of the Markov inequality, J. Approx. Theory (submitted).

2. P. Erdös, An extremum-problem concerning trigonometric polynomials, Acta Sci. Math. Szeged 9 (1939), 113-115.

3. T. J. Rivlin, The Chebyshev polynomials, Wiley, New York, 1974.

4. J. Szabados, On some extremum problems for polynomials, Proc. Conf. Approximation and Function Spaces (Gdansk 1979), PWN, Warsaw (to appear).

Department of Mathematics, University of Sofia, 1126 Sofia, Bulgaria 